\title{
Remote Monitoring of Heart Patients Using Robotic Process Automation (RPA)
}

\author{
Shwetha $\mathrm{R}^{1 *}$, and Dr. Kirubanand V B \\ ${ }^{1}$ Department of Computer Science - CHRIST (Deemed to be University), Bengaluru, India
}

\begin{abstract}
After the age of 60 years or older many people are diagnosed with heart related issues to follow up older patients, bedridden, who needs maximum care at their homes implementation of phone monitoring becomes necessary. According to study many heart patients are supposed to have a regular check up to keep a track on their physiological data. Health care is a ground that is rapidly developing in services and technology. A recent development in this region is remote monitoring of patients which has many benefits in a fast-aging world population with increasing health complications. Sensors are used to monitor essentials or vital requirements such as heartbeat, blood pressure, temperature, blood glucose level and many more. Remote monitoring varies for every age group and every aspect. During pandemic times its difficult for many of them to move around, thus remote monitoring of patients helps to have a safer and efficient way to monitor them and also saves time. These new technologies can make a contact-less and monitor illness based on the sensor values. To make remote monitoring more efficient automation places a vital role. In health sector, to make any process automated RPA (Robotic process automation) is used. Using the established application software robots automate the process originally performed by human beings. This paper focuses on easy way to monitor patients who are in a distant place, have a regular check-up without visiting the doctor regularly and in case of emergency contact the doctor within no time.
\end{abstract}

\section{Introduction}

The term heart disease refers to a range of conditions which affect our heart and makes a lot of variations in our body. It varies according to the age groups. Common symptoms are chest pain, shortness of breath, numbness, or coldness in legs or arms, discomfort and nausea and many more. 4 out of 5 people who are 70 years and older die due to heart attack. All these comes under CVD (Cardiovascular diseases). Even though advanced treatments are available in the health care, due to certain conditions and lifestyles of many people Cardiovascular diseases are increasing rapidly. Few causes of these diseases are diet, body mass, stress, anxiety medication side effects genetic disorders. Diet: There are good cholesterol and bad cholesterol we need good cholesterol to our body such as HDL with a good count of HDL it lowers the risk of heart related issues, LDL and VLDL are the bad cholesterol which causes major blockages in the valves.

It can be a by-product of what we consume which accumulates in our body and it can be seen in our increasing weights. This can be due to due to bad food habits and drinking habits. Obesity is a condition which accumulates bad cholesterol and makes us vulnerable to heart diseases. It is defined by the World Health Organization as unnecessary fat that accumulate and present a risk to health. Obesity is measured in body mass index (BMI), which is a person's weight (in kilograms) divided by the square of his or her height (in meters). Weight (in kilograms) divided by the square of his or her height (in meters). A person with a BMI of 30 or more is generally considered obese. A person with a BMI equal to or more than 25 is measured over weight $[1,2]$. Rest of the issues such as anxiety, side effects are inevitable. Most of these causes can be diminished with a simple change in lifestyle.

\subsection{Remote Patient Monitoring}

Remote monitoring and automation is a process that can help reduce some of the burden off of the network administrators. It keeps a track on all the activities. Automation software works more efficiently than a human being. This improves overall efficiency and maximizes their up time. It is an extremely nonintrusive way to gather data and information about patients. In a pandemic situation like covid-19 it is difficult to go to a check-up regularly and spend more time on it. The patients who are just discharged after a heart surgery will have to get a check-up regularly, this automated remote monitoring will be useful to those patients which helps in monitoring our heart rate with a wearable sensor and analyse the blood pressure and notify the doctors if there are any abnormalities. This reduces time and also it is safe as the patient can get a quick check up in their desired location. 


\subsection{Why RPA?}

Robotic process automation is the only automation technique used in the medical field. This is more reliable and gives more accurate results. Human errors can be reduced and thus has more accuracy. This can save a lot of time and during any major emergencies it can notify the doctors early can possibly save a person's life. It accelerates time to value it where traditional way takes few days create test and deploy. RPA takes just few hours. Increases the throughput. This way we can experience more and better outputs in less time. RPA software can manipulate collected data to generate analytic that offer clinical staff valuable insights to help them make more accurate diagnoses and offer tailored treatments to patients. This method is only for a regular and basic check-up such as monitoring the pulse rate and blood glucose level. In case any variation in these parameters' patient can be sent to hospitals on doctors' advice. This saves a lot of time for both doctors and patients. This is a better approach than the traditional method. In a pandemic situation it is always better not to go out much. This helps the patient to get a quick check up and the doctors will be notified if there is any abnormalities found.

Table 1.RPA Process

\begin{tabular}{|c|c|c|c|c|}
\hline $\begin{array}{c}\text { Front } \\
\text { Office }\end{array}$ & $\begin{array}{c}\text { Middle } \\
\text { Office }\end{array}$ & $\begin{array}{c}\text { Back } \\
\text { Office }\end{array}$ & $\begin{array}{c}\text { Regulatory } \\
\text { Compliance }\end{array}$ & $\begin{array}{c}\text { Data } \\
\text { Intelligence }\end{array}$ \\
\hline
\end{tabular}

Primary Objective of this paper is to introduce RPA in remote monitoring. It acts as an add-on to an existing model; RPA is used major in Health Sectors. It can automate our anti-virus solution, patch updates, backup process and create tickets through the web portal that will notify you if something is awry. Using RPA, health care companies can automate the check-in process for patients by using self-service terminals integrated into the facility's database. The patient's conditions could also contribute to how much of a priority they are, this would allow the RPA software to automatically organize the list of patients by priority as they entered the system. Automation helps in reducing risks and gives better accurate results and it diminishes a lot human error.

\section{Literature Review}

There are many different ways in managing a patient and monitor them remotely. All the techniques are mainly focused on how to make use of technology well and help patients in early detection and prevention of worsening of illnesses. This has a lot of advantages for both patients and hospitals. This can reduce the resources usage and also this is efficient way to keep a check on recently discharged patients. These are the main objective of remote monitoring.

Remote monitoring makes more sense for the patients who are recently discharged.

The management of patients with prolonged heart failure (HF) induces a high burden on health care resources due to the regular review, visits combined with repeated hospitalizations due to cardiac decomposition.[2] Early identification of $\mathrm{HF}$ deterioration is crucial to prevent HF-related hospitalizations, potentially improve overall survival and quality of life and lower the burden on health care resources. Remote monitoring of chronic HF patients can aid in the detection of HF weakening; therefore, several remote monitoring strategies have been developed.[3]A recently updated Cochran's review included 41 randomized controlled trials (RCTs) investigating the use of structured telephone support or non-invasive tele-monitoring compared with standard HF care [3]. This review helped in getting a into a model where we can make the process of remote monitoring more efficient. As most of them prefer a tele-monitoring first. This increases the growth of the automation of this process too.

With the help of wearable sensors, the patient can test his heart rate and blood pressure by himself. Multiple large multicentre prospective clinical studies and RCTs have examined multiple non-invasive remote monitoring strategies, ranging from indication and body weight monitoring to complex and intensive strategies including body weight, blood pressure, electrocardiography and peripheral capillary oxygen saturation. Standalone systems allow patients, usually in their own homes, to send non-invasively measured data to their health care team, by either telephonybased systems or the internet. Heart rate variability (HRV) discusses the variation (over time) in the period concerning the consecutive heartbeats in a person, which reflects his physiological signs that modulate his heart rhythm. HRV is an indication of the current heart-related abnormalities and can warn about the imminent cardiac diseases.

[4] RPM via regularly scheduled structured telephone contact between patients and Doctors/health care providers or electronic transfer of physiological data using remote access technology via remote external, wearable, or implantable electronic devices is a growing modality to manage patients with chronic HF. [7] Here the electronic transfer of data made more. Various technologies such as actual ECG monitors or textile- based wearable systems are used to get these data. Remote monitoring represents a novel intensified follow-up strategy of heart failure management.[6] The doctors can monitor the deterioration or maintenance of the patient while also advising the paramedics who are physically with the patients as necessary. Doctors can work on two consecutive things and attend more patients. An application for Android OS, which is widely applied in portable devices all over the world, was developed by our team. Its main tasks include data processing reporting of test results to the user test scheduling during the day dialling of automatic emergency calls or sending an SMS in case of critical user conditions [5] this application also provides controls and Bluetooth connections for easy access and report generation for the patients.

This method helps in monitoring elderly and patients with heart issues. Mobile Application is easier 
to handle. The engineering varies from sensors attached to body to sensors attached to the environment and new innovations allow contact less monitoring which requires only the patient to be present within a few meters from the sensor. System can measure body temperature, ECG, saturation levels, air flow in lungs oxygen, and galvanic skin response. [10] Personalized algorithms are used to make the predictions equal to the expectations. Although majorly to detect abnormalities threshold algorithms are used. AI in health care include implementing ML approaches to track individual patient health, deploying advanced analytic to scale-invariant feature transform through large data sets to realize clinical skillfulness.[9] In all the above-mentioned papers authors have made a survey on couple of patients and given their results. All the RPM (remote patient monitoring) techniques here focuses on early detection. This is easier for recently discharged patients, giving them a quick check up once a day and reporting to the doctor instantly. RPA is more accurate than human workers and runs at a higher throughput. They can greatly benefit from RPA thanks to its advantages which could be even more powerful if machine learning is applied to the RPA processes.

\subsection{Existing models}

All the existing models have their own add-on to this method, each method helps patient analyse their reports this also keeps a transparency between doctors and patients. The most suitable method to perform our implementation was using wearable sensors and web application to show the data and send it to doctors as well. This method consists of wearable sensor and a device and a web server. Here patient can wear sensor and check his heart rate or pulse rate and that data will be stored in the web application. The data stored will be sent immediately to the doctors and notify the details of the patient. This method transmits real-time data wireless from wearable devices worn by the patient to second tier of the system via Bluetooth low energy. Zephyr BT is a wearable sensor which has been used to extract heart rate information of the patients requiring continuous monitoring.

As this consists of low energy, we need not worry about the other technical issues such as battery and usage. This records the heart rate of the patient at that particular time and stores that information in a web application this can be done in an android application as well. To even check the progress this application can be used. It can keep a track on everyday status and this gives a clear idea to patient to diagnose themselves. Many Machine Learning algorithms are implemented to get better accuracy and get better predictions. Each technique follows a pattern and a different algorithm. Similarly, many methods have been implemented so far and have used threshold algorithms in order to have a track on the patient heart beat or pulse rate. Similarly, many methods have been implemented so far and have used threshold algorithms in order to have a track on the patient heart beat or pulse rate. This way we can monitor heartbeat of recently discharged patients and follow the instructions of the doctor on a phone call that is using tele-monitoring system. Below mentioned are few models which works similarly to our proposed method.

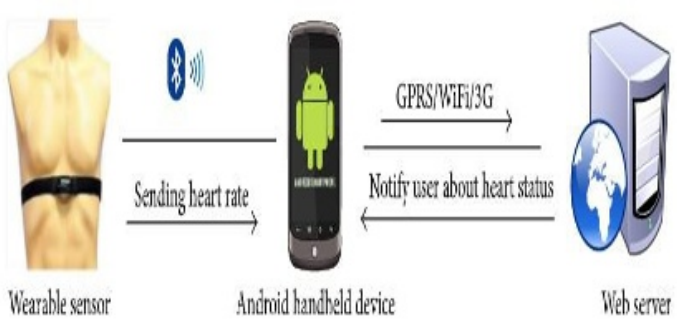

Fig. 2. Working of existing model-1

Above Figure- 2 shows the working of how this method works, it is reading the values using sensor and sending heart rate using Bluetooth and that value is sent to web.

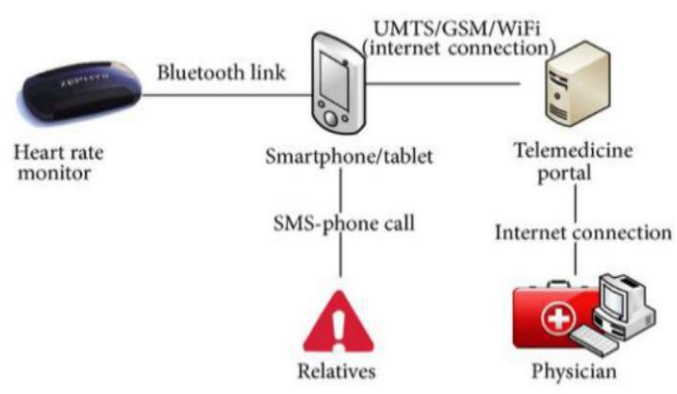

Fig. 3. Working of existing model-2

The working sequence of the existing model 2 is given in Figure 3. This is one more method where RPA can be introduced even in this technique patients heart rate is recorded and that data is stored in smart phones. Using telemedicine portal, a doctor will be notified. Here Heart rate monitor is again a sensor which is linked to a phone using Bluetooth the data will be printed. This is exactly how it works in smart watch where it takes data from human body based on their activity and data will be displayed in a format on a mobile application. Both the systems are based on a simple, inexpensive, and wearable device whose functionalities are comparable to those of more expensive and sophisticated clinical devices.

\section{Proposed Work}

\subsection{Data Collection}

This Data set is a real time sensor data which consists of 120 patients diagnosed of heart conditions based on various medical factors such as Blood Pressure, Temperature, Pulse, Glucose level. The attributes in the data set are Patient ID, Patient Name, Gender, Age, Temperature, Blood Pressure, Pulse, Blood Glucose and Heart Rate. Many operations were performed on this data set. Initially a co-relation of each feature in data set was calculated. It was found that heart rate, 
temperature and blood glucose level are co-related. The co relation explains these values are directly proportional. When Temperature increases the Heart Rate, temperature and Blood Pressure also increases.

These indications help to analyse the data set better and perform functions on it.

KNN algorithm is used to set target classes which are approximately of same size. $\mathrm{KNN}$ is the most efficient algorithm used for pattern recognition. Using this algorithm, we are estimating if the person is suffering from any abnormalities or not. This was done based on the target classes which was male and female. While processing this data, converting few explicit variables into dummy variables and scale all the values before training the ML models are necessary.

$\mathrm{KNN}$ model was trained and different $\mathrm{K}$ values and the scores with $\mathrm{K}$ neighbors and different $\mathrm{K}$ values were recorded. This helps in understanding the prediction of heart issues on that particular patient. Further on this Robotic Process automation implementations and its working can be seen. This prediction can help in a lot of ways we can even increase the value of $\mathrm{K}$ and try it on $\mathrm{N}$ values which is gives more understanding on it. The graph below is the representation of $\mathrm{K}$ Neighbours Classifier scores for different $\mathrm{K}$ values.

With the resultant data of the trained KNN model, we will be estimating the extent of the threat when it exceeds the threshold value, the Doctors, Patients or caretakers will be notified of the diagnosed health condition instantly thus reducing the fatality rate because of negligence or delay in treatment protocol.

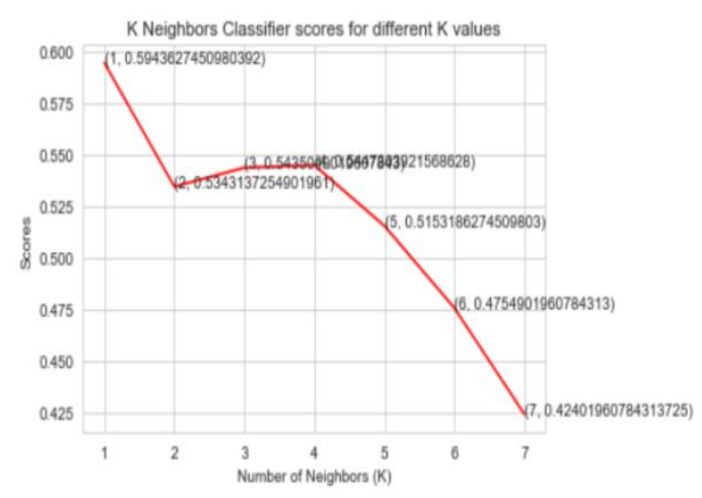

Fig. 1. Representation of K Neighbours Classifier scores for different $\mathrm{K}$ values.

\section{Implementation}

After collecting suitable existing models, Automation can be implemented very easily on web applications or mobile applications. Thus, using wearable sensors data should be collected and the values will be displayed in a web application/excel and those values will run in BOT using UiPath. In this we can collect multiple patient data and check which patient has what abnormalities, we can consider any attribute such as temperature, blood pressure and heart rate. Here we are using BOT to automate the process of getting data and comparing it and sending out the alters. ID of every patient will be unique thus we can search for a particular patient. Most of the work will be done by BOT. It sorts the data and sends the alerts when there were any abnormalities found.

To check for abnormalities and send out triggers we are setting a threshold value and comparing that threshold value with the BOT and send a notification regarding the abnormalities, we can fetch the criteria and perform operation on it. Figure 4 and Figure 5 represents the sample data which consists of criteria such as Heart rate, Blood Pressure, Temperature. Here which ever factor is higher than the threshold value that data will be displayed. In this we have considered heart rate. In the Fig. 4 it shows that heart rate is high we can specify the patient ID and display an alert saying Patient 1234 Heart rate is high. This can be done for multiple values at a time. This method helps the doctor to attend multiple patients at a time.

The same process was performed on the sensor dataset which has reports of 120 patients as mentioned earlier. The same BOT runs and checks for all the 120 patients heart rate and other attributes and whichever value is higher than the threshold value those value will be stored in a new file. This is the file which doctor gets to see first and can immediately contact the patient. Here RPA is automating the process of manual general check-ups for a patient. Certain examples where BOT shows a notification.

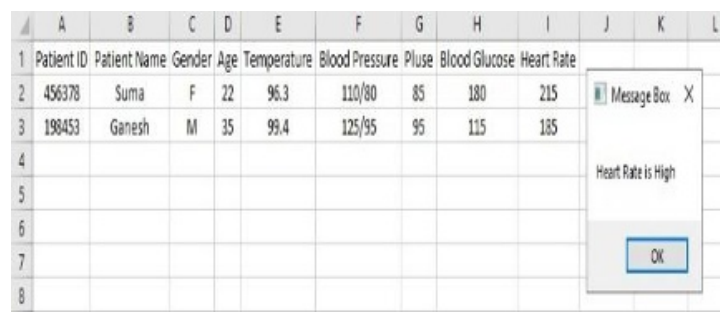

Fig. 4. High heart rate notification.

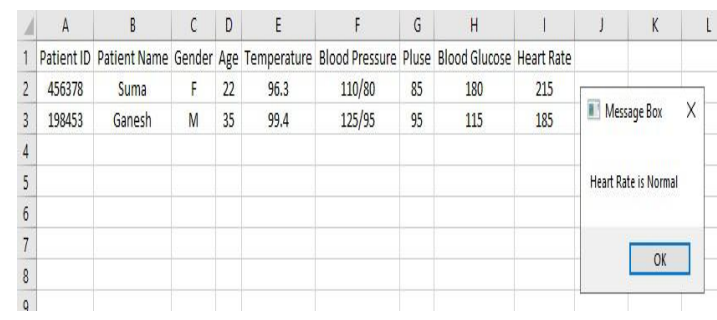

Fig. 5. Normal heart rate notification.

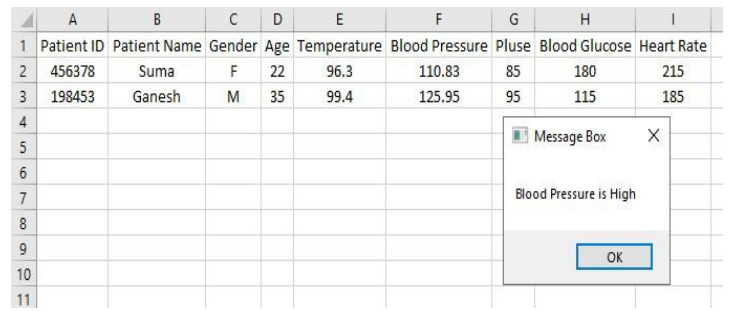

Fig. 6. High Blood Pressure notification. 


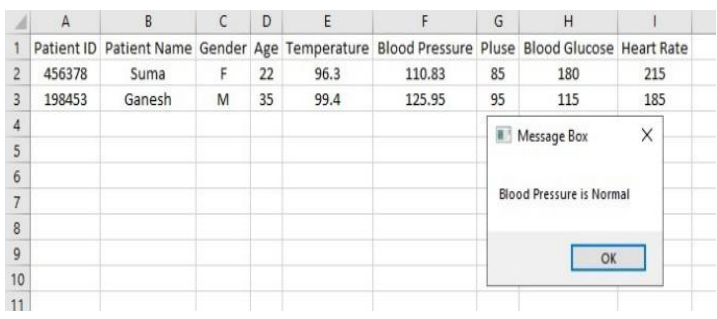

Fig. 7. Normal Blood Pressure Notification.

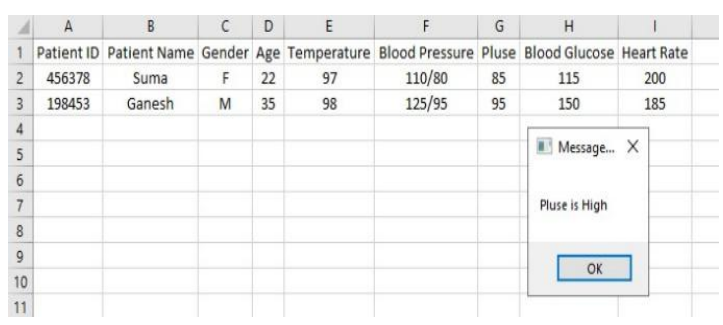

Fig. 8. High Pulse Notification

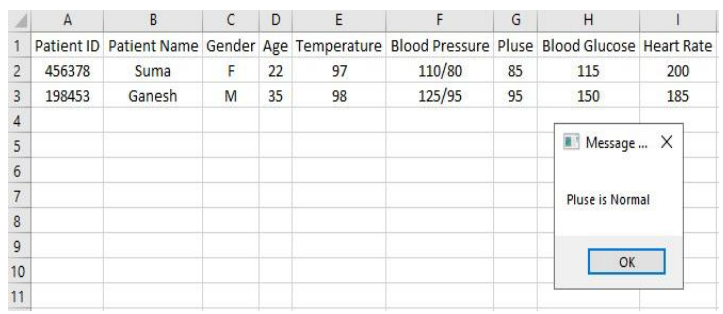

Fig. 9. Normal Pulse Rate Notification

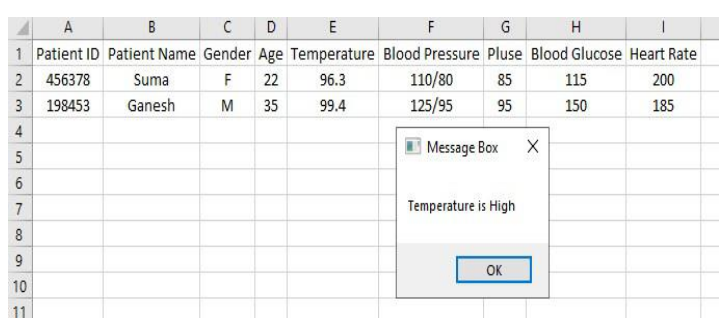

Fig. 10. High Temperature Notification

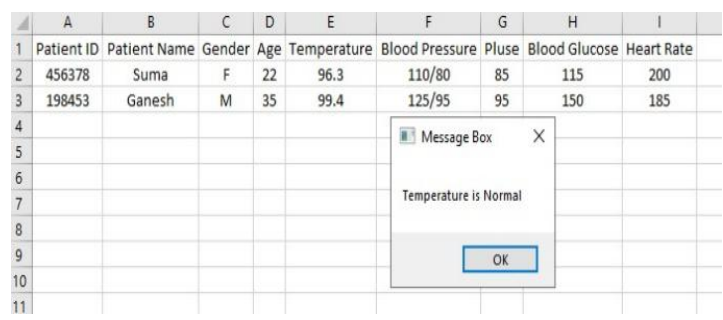

Fig. 11. Normal Temperature Notification

The above given images in Figure 4, 5, 6, 7, 8, 9, 10 and 11 were only for lesser number of patients. Same implementation was applied to a larger number of patients.

\section{Results}

If any of the values are greater than the threshold value, the comment," Please check on the patient records." will be added by the records. This helps the doctor to contact the patient immediately. The data set is processed and the output will be stored in a different file where only the values greater the threshold value will appear. Age and Heart Rate goes hand in hand.

Result Graph represents the age and Heart rate. The person is at more risk with the increasing in age. Age is directly proportional to heart rate.

\section{RESULT}

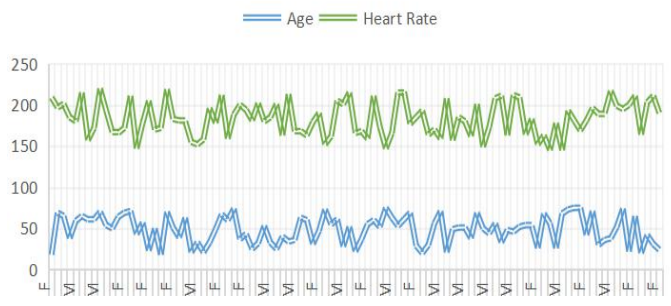

\section{Fig.12. Result Graph}

RPA is implemented on most of the business and it is more actively used tool. Many articles have been published about the usage of RPA in health care sectors. RPA in health care results in tracking and tracing many standardized, patient specific routes. It enhances the data confidentiality as well.

\subsection{Advantages and Disadvantages}

There are many advantages in this, Robotic Process Automation helps in faster and better execution and also gives more accurate results. It can be considered as an evolved version of present system. Implementing RPA on present or existing methods save a lot of time and money. It provides a better and efficient way to keep a track on the patient details. Here there is no need of any manual entry of the details so it also reduces human errors. This is very helpful for busy doctors who can just take a quick glance of multiple patients at a time and have a telecommunication mode soon. As far as patient is concerned, he can go to doctor only when there is abnormalities and only when doctor contacts them. This way we can save time and help elderly or mentally challenged people to have a treatment at home also get a good monitoring system. This reduces a lot of risks.

Disadvantages can be regarding signals and bandwidth patients should always have a stable internet connection and should also take care of their sensors and their batteries, any fault in any of these may result in a wrong output. This can again put the patient at risk in case of emergency.

\subsection{Comparison with traditional and automation methods}

With traditional method only one task can be performed at a time. For instance, only one patient data can be seen and the doctor should manually check for abnormal entries. But, using RPA we can perform multiple tasks at a time. All the process can go hand in hand without any disturbances. Doctors can prioritize the patient list according to the alerts available. Doctors can check many patient data at once and the 
check-up will be done much faster than the usual check-ups. patients need not wait for a long period of time. In traditional methods each patient to should visit the doctor and get a clarification, this is very risky during pandemic like covid-19. And also, patient has to go through many other safety measures before getting into hospital. Instead for all the general check-ups patient can do it at home with these methods and doctors can notify if there is emergency.

\section{Conclusion}

This research work concludes that Application of Robotic Process Automation helps the patients and doctors to get a report sooner and also reduces time to travel to hospital each time for general check-up. Instead, they can go to doctor only when it's necessary. This method is cost efficient and accurate compared to other traditional methods.

RPA check that all process steps are traceable, documented and tracked, as well as consistently organized in well-structured log offers a quick solution. Software robots can provide very much needed helping hand to the industry facing new requirement as times are constantly changing.

\section{Acknowledgement}

The author honestly thank the research guide for their continued support and motivation.

\section{References}

1. Priyanka Kakria, "A Real-Time Health Monitoring System for Remote Cardiac Patients Using Smartphone and Wearable Sensors, "Article Volume 2015.

2. Maurizio Volterrani, Barbara Sposato, Remote monitoring and telemedicine, European Heart Journal Supplements, Volume 21, Issue Supplement, 1 December 2019

3. Brahmbhatt, Darshan Cowie, Martin. (2019). Remote Management of Heart Failure: An Overview of Telemonitoring Technologies. Cardiac Failure Review. 5. 86-92. 10.15420/cfr.2019.5.3.

4. A. F. Hussein, N. A. kumar, M. BurbanoFernandez, G. Ram 'irez- Gonza'lez, E. Abdulhay and V. H. C. De Albuquerque," An Auto- mated Remote Cloud-Based Heart Rate Variability Monitoring System," in IEEE Access, vol. 6, pp. 77055-77064, 2018, doi: 10.1109/ACCESS.2018.2831209.

5. An Android-Based Heart Monitoring System for the Elderly and for Patients with Heart Disease Paola Pierleoni ,1 Luca Pernini ,1 Alberto Belli,1 and Lorenzo Palma 1 Dipartimento di Ingegneria dell'Informazione, Universita' Politecnica delle Marche, Via Brecce Bianche, 60131 Ancona, Italy Aug 2014
6. Mihran Martirosyan, Kadir Caliskan, Dominic A.M.J. Theuns Tamas Szili-Torok (2017) Remote monitoring of heart failure: benefits for therapeutic decision making, Expert Review of Cardiovascular Therapy, 15:7, 503- 515, DOI: 10.1080/14779072.2017.1348229

7. A Meta-Analysis of Remote Monitoring of Heart Failure Patients, Journal of the American College of Cardiology, Volume 54, Issue 18, 2009

8. Andrew Steger "What does robotic process automation look like in Health care" HealthTech magazine January 2020

9. Developing an Artificial Intelligence-Enabled Health Care Practice: Rewiring Health Care Professions for Better Care Author links open overlay panel David Wiljer MA, PhD ZakiHakim MSc, MHIa November 2019.

10. Remote patient monitoring: a comprehensive study Lakmini P. Malas inghe1 - Naeem Ramzan1 . Keshav Dahal1 